\title{
Peace of Mind Program (PMP): Impact of the Dissemination and Implementation of an Evidence- based Intervention (EBI) to Improve Mammography Appointment Adherence in Safety Net Clinics
}

Jennifer Holcomb ( $\nabla$ jennifer.I.holcomb@uth.tmc.edu )

The University of Texas Health Science Center at Houston School of Public Health https://orcid.org/0000-0001-7226-7642

\section{Suja S Rajan}

The University of Texas Health Science Center at Houston School of Public Health

\section{Gayla M Ferguson}

The University of Texas Health Science Center at Houston School of Public Health Jiali Sun

The University of Texas Health Science Center at Houston School of Public Health

\section{Gretchen H Walton}

The University of Texas Health Science Center at Houston School of Public Health

\section{Linda Highfield}

The University of Texas Health Science Center at Houston School of Public Health

\section{Research}

Keywords: Breast cancer screening, underserved women, evidence-based intervention, patient navigation, mammography adherence, implementation science, Consolidated Framework for Implementation Research, safety net clinics

Posted Date: May 11th, 2021

DOI: https://doi.org/10.21203/rs.3.rs-493474/v1

License: (c) (1) This work is licensed under a Creative Commons Attribution 4.0 International License. Read Full License 


\section{Abstract}

\section{Background}

he Peace of Mind Program - an adapted evidence-based intervention to improve mammography appointment adherence in underserved women - was expanded to safety net clinics. This study assessed effectiveness of the intervention in improving mammography appointment adherence and implementation of the intervention.

\section{Methods}

The intervention was implemented through a non-randomized stepped wedge cluster design with 19 Federally Qualified Health Centers and charity care clinics in the Greater Houston area. Clinics were their own control during the baseline period and conducted at least three mammography drives during the baseline and intervention period. A multivariable generalized estimating equation logistic regression was conducted to examine mammography appointment adherence. To examine adoption and implementation of the intervention, two surveys assessing Consolidated Framework for Implementation Research constructs were conducted with clinic leadership and staff. One-sided t-tests were conducted to analyze mean score changes between the adoption and implementation survey.

Results

total of 4402 women (baseline period $=2078$; intervention period $=2324$ ) were included in the final analysis. Women in the intervention period were more likely to attend or reschedule their mammography appointment $(O R=1.30 ; p<0.01)$. Similarly, for those in the intervention period, women who completed the intervention were more likely to attend or reschedule their mammography appointment than those who did not complete the intervention $(\mathrm{OR}=1.62 ; \mathrm{p}<0.01)$. The mammography appointment no-show rates for those in the baseline period, in the intervention period, and who completed the intervention were, respectively, $22 \%, 19 \%$, and $15 \%$. In terms of the adoption and implementation survey, a statistically significant mean score decrease was observed in Inner Setting overall and in two Inner Setting constructs, Culture - Effort and Implementation Climate.

\section{Conclusions}

This study provided a pragmatic approach to translating an evidence-based mammography intervention into practice in safety net clinics. While the intervention improved mammography appointment adherence, there our opportunities to further integrate Consolidated Framework for Implementation Research constructs in future implementation of the intervention. Future research on the effects of implementation moderators particularly Inner Setting constructs would be of value to implementation practitioners.

\section{Contributions To The Literature}


- The findings address an existing research to practice gap in understanding effective implementation and dissemination of evidence-based cancer screening interventions.

- Utilizing adoption strategies, identifying clinic readiness, capacity for implementation, and providing implementation strategies to support clinics are important to promote the successful uptake of an evidence-based intervention in safety net clinics.

- While previous research has shown the importance of the assessment of Consolidated Framework for Implementation Research constructs to better understand and improve implementation, this study highlighted challenges in the timing of the assessment during implementation, engagement of clinic staff in the assessment, and understanding effects of constructs on implementation.

\section{Background}

Breast cancer remains the second leading cause of cancer related death among women in the United States, with one in every eight women developing breast cancer in their lifetime. ${ }^{1}$ Breast cancer-related health disparities exist for underserved women and especially for Black women leading to increased mortality and morbidity. ${ }^{2-3}$ Numerous biological, social, and environmental factors contribute to breast cancer- related health disparities in underserved women, but routine mammography screening adherence remains the key predictor of disease survival and reduction of these disparities. ${ }^{4-8}$ Research has found seasonality, geographic location, lack of knowledge of familial history of breast cancer, racial discrimination, lack of trust of healthcare providers, lack of primary care physician, lack of access to healthcare, cultural support systems, religious beliefs, lack of knowledge, conflicting priorities, and lack of transportation to be drivers of mammography screening non-adherence. ${ }^{5-10}$ Mammography screening appointment adherence can be a critical component to receiving an early stage breast cancer diagnosis. ${ }^{11}$ Missing one mammography screening increases a woman's likelihood of developing a late stage breast cancer. ${ }^{5,12-14}$ As mortality and morbidity increases with breast cancer stage, it is critical to diagnose breast cancer in an early stage. ${ }^{15}$ Underserved women are at higher risk for late-stage diagnosis due to lower mammography screening rates, missed or no-show appointments, and increased time between referral, diagnostic examination and treatment. ${ }^{16-21} \mathrm{~A}$ lack of healthcare access might prevent underserved women from receiving a recommendation for a mammography screening. ${ }^{10}$ Understanding and addressing barriers to mammography appointment adherence in women who are not able to receive routine mammography screenings is the best way to reduce health disparities. Mammography screening interventions addressing both the structural (transportation and cost) and psychosocial (fear of cancer detection) barriers can promote appointment adherence in underserved communities. ${ }^{20-21}$ Patient navigation and counseling programs and evidence-based interventions (EBIs) addressing these same barriers have demonstrated success in increasing mammography appointment adherence. ${ }^{20-23}$ Although mammography screening interventions might alter mammography screening behavior, in order to be successful, interventions should be adapted and tailored to meet the unique needs of the proposed communities of interest. ${ }^{21-23}$ 
For the success of EBls to be translated to different communities, researchers with implementation knowledge must work collaboratively with community and clinical stakeholders in intervention adoption and implementation. ${ }^{24-25}$ An example of a clinical stakeholder which can help tailor interventions are mobile mammography providers who can provide screening at no or low cost at clinics close to the underserved communities and neighborhoods, helping to eliminate geographic, cost and transportation barriers. ${ }^{20}$ In addition, dissemination and implementation (D\&I) practitioners aiming to implement a tailored intervention to intercede across both cost and access to care among underserved communities have partnered with safety net healthcare systems and Federally Qualified Health Centers (FQHC). FQHCs are community-based health care providers that provide culturally competent primary care and preventative services in underserved communities. ${ }^{21}$ The nature of FQHCs lends them direct access to underserved women most likely in need of mammography screening services, but also creates barriers to adoption and implementation of an EBI. ${ }^{26-27}$ There is a gap in understanding of effective D\&I methods in successful interventions, particularly those aimed at cancer prevention and detection. ${ }^{28}$

The current study utilized a theoretically based, D\&I strategy to facilitate the implementation of an evidence-based mammography screening intervention focused on reducing mammography screening non-adherence in underserved women with FQHCs and charity care clinics and mobile mammography providers. The Peace of Mind Program (PMP) intervention is an evidence-based mammography screening intervention based on the Transtheoretical Model of Change adapted from a research tested intervention program from the National Cancer Institute's Research Tested Intervention Programs (RTIPs) database. ${ }^{28-29}$ In the current study, the PMP intervention was expanded to include implementation components geared toward supporting implementation in safety net clinics, and by doing so, aimed to address the research to practice gap. ${ }^{28-29}$ We hypothesized that the PMP intervention would improve mammography appointment adherence in underserved women compared to usual care. The study objectives were targeted to test the effectiveness of the PMP intervention in improving mammography appointment adherence and to assess implementation of the PMP intervention [See Additional file 1: Standards for Reporting Implementation Studies (StaRI) checklist].

\section{Methods}

\section{Intervention}

The Peace of Mind Program (PMP) is an active listening, tailored telephone reminder call intervention to counsel women through barriers to mammography screening appointment attendance. ${ }^{28-29}$ Each woman during the intervention period received up to three reminder call attempts for their scheduled mammography screening appointment at a clinical site. If the woman did not answer on the first attempt, two additional attempts were made to reach the woman. If the woman answered the phone call, but did not consent to participate in the study, she was reminded about her appointment in the usual care manner of each site. If she answered and consented to participate, a Certified Community Health Worker $(\mathrm{CHW})$ who made the call assessed the woman's confidence in attending their scheduled mammography 
appointment, counseled the woman through barriers to attending the appointment, and recorded the woman's responses in an online interface program designed in RedCap. The results of each reminder phone call, whether the call was completed or a message left, and the woman's resulting mammography appointment attendance or no-show status were also recorded in RedCap. RedCap served as the online platform for both data storage and initial data analysis for this study.

\section{Implementation Strategy}

Prior to implementation of PMP, potential clinics were engaged through various adoption strategies including adoption meetings, webinars, and an adoption survey measuring Consolidated Framework for Implementation Research (CFIR) constructs across multiple domains. Those clinics who adopted the program then participated in several implementation strategies, including: stakeholder meetings, a continuing education unit (CEU) certified 2-day training for clinic community health workers (CHWs), and a site readiness assessment checklist to prepare for implementation. In addition, the community partner - the Breast Health Collaborative of Texas (BHCTexas) - and research team worked with participating clinics to align goals for mobile mammography drives and facilitated relationships with mobile providers as needed to support implementation. Onsite role modeling and support from BHCTexas CHWs and ongoing technical support from the research team was provided throughout PMP intervention implementation. Financial resources included a $\$ 7500$ stipend for clinic participation and financial assistance for women to receive free screening. At eight weeks post-implementation, clinics were invited to complete an implementation survey to assess the same constructs as the adoption survey. A full description of the development, adoption, implementation, and stakeholder engagement components of the PMP intervention has been reported elsewhere. ${ }^{28-30}$

\section{Study design}

A non-randomized stepped wedge cluster design was used to assign clinics into two non-concurrent implementation waves with two to three groups in each wave. ${ }^{28}$ In order to participate in PMP, clinics must: (1) have been members of BHCTexas within the Greater Houston service area, (2) had a designation as a FQHC by the Health Resources and Services Administration (HRSA) or be a charity clinic which provides free or reduced cost care to underserved populations in their service area, (3) serve women between the ages of 40 to 64 years old who were at or below $200 \%$ of the federal poverty level for a family of four and who lacked health insurance, and (4) engage in provision of mammography screening services at least six times per year (three in baseline; three in intervention) and (5) women at the clinic must have been in need of mammography screening and be scheduled for an upcoming appointment. Patients must have completed a clinical breast exam prior to their scheduled screening appointment per mobile provider requirements. Variation across clinics existed in frequency of mammography drives, number of patients scheduled in each drive, number of staff available to participate in PMP, existing relationships with mobile mammography providers, and available funding and resources which resulted in differences in clinic readiness to start the PMP intervention. Due to these differences, the randomized allocation of the clinics into waves and groups as previously described was 
not possible. Clinics that had lower levels of readiness were assigned to later groups to benefit from more time to receive implementation strategies from PMP. Each clinic served as its own control during the baseline period and was required to have at least three mammography drives during both the baseline and intervention periods. Clinic, BHCTexas, and research team staff were not blinded in the study. Since each clinic served as its own control during the baseline period, blinding was not possible or necessary for this study.

\section{Dependent variable}

The dependent variable was a dichotomous variable indicating mammography appointment adherence with a no-show or cancelled appointment categorized as "0" and an attended or rescheduled appointment categorized as " 1. "

\section{Independent variables}

The independent variables of interest are two dichotomous variables indicating study period, baseline period (categorized as "0") or intervention period (categorized as "1") and for the intervention period, whether the patient did not complete (categorized as " 0 ") or did complete (categorized as " 1 ") the PMP intervention. A patient completed the intervention if they answered the reminder call, consented to the study, received the staging question assessing confidence and barriers counseling. In addition, patient, intervention, and clinic variables examined in the analysis of the data included age, season, wave, group, mobile mammography provider, and clinic racial/ethnic distribution. Age was categorized into three age groups - 55 and above, 45 to 54 and 25 to 44 years - to align with the age-based mammography screening guidelines. The season in which the patient scheduled their appointment was categorized by winter (January to March), spring (April - June), summer (July to September), and fall (October to December) to examine a possible seasonal effect. ${ }^{31-32}$ The wave was categorized as a dichotomous variable (0/1) for wave 1 and 2 and the group was categorized as a three-category variable (group 1, 2, and 3) based on when the clinic began the PMP intervention. Each of the three mobile mammography providers were categorized as a dichotomous variable based on if the provider assisted with mammography screenings at the clinic in which the patient was scheduled (categorized as " 1 ") or not scheduled (categorized as "0") for a mammography appointment. The provider with an existing reminder call and group education program for usual care was defined as the reference group. The clinic racial/ethnicity distribution (percentage) of the population served by each clinic from 2015 to 2016 was collected from the Health Resources and Services Administration (HRSA) Uniform Data System (UDS) for FQHCs and the Texas Association of Community Health Centers (TACHC) for charity care clinics. The clinics were categorized based on the racial/ethnicity group with the highest percentage in five mutually exclusive groups: non-Hispanic Black, non-Hispanic white, non-Hispanic other (another race other than Black or white), Hispanic, and multi-racial/ethnicity group (equal percentage of non-Hispanic Black, nonHispanic other, and Hispanic women served). Each of the five racial/ethnicity groups were also categorized as a dichotomous variable indicating if the racial/ethnicity group was the highest reported for the clinic (categorized as "1") or not the highest (categorized as "0"). 
To assess fidelity of PMP intervention implementation, implementation variables examined in the analysis of the data for those in the intervention period, included the CHW who made the appointment reminder call, if the patient answered the reminder call, the number of reminder call attempts made by the $\mathrm{CHW}$, and language in which the reminder call was received. The $\mathrm{CHW}$ who made the reminder call was a dichotomous variable based on if they were a clinic staff member (categorized as "0") or a BHCTexas staff member (categorized as "1"). A dichotomous variable determined if the patient answered the reminder call (categorized as " 1 ") or not (categorized as " 0 "). The number of reminder call attempts received by patient in the intervention period was a dichotomous variable for one call (categorized as " 0 ") or multiple call attempts (two or three calls categorized as "1"). Language was categorized by English, Spanish, or Vietnamese.

\section{Data analysis}

A descriptive analysis was performed to examine differences in patient, intervention, clinic, and implementation variables across the baseline and intervention periods. To test for statistically significant differences in mammography appointment adherence across the baseline period, intervention period, and covariate variables we used chi-square tests. For the primary analysis, we used a multivariable generalized estimating equation (GEE) regression model to examine mammography appointment adherence in two analytical models. In the first model, we included all patients in the baseline and intervention period (intent to treat analysis). In the second model, we included only patients in the intervention period to examine those who did and did not complete the intervention (i.e., completed the reminder phone call). We modeled clustering across the 19 clinical sites using a logistic GEE regression (logit link with odds ratio) and an independent correlation structure. We analyzed age, season, wave, group, mobile mammography provider, the group variable and the five dichotomous variables for clinic racial/ethnic distribution, and for those in the intervention period, the $\mathrm{CHW}$ who made the appointment reminder call, if the patient answered the call, the number of reminder call attempts, and language independently in each of the models and added each additional variable as a covariate. The Quasi-Akaike information criterion (QIC) value was used to identity covariates to include in the final models. All analysis was performed using Stata 14.0 (College Station, TX) with $\mathrm{a}=0.05$ as the limit for statistical significance.

To measure adoption and implementation factors associated with each clinic, a survey was conducted with clinic leadership and staff with any potential role in PMP prior to adoption of PMP and eight weeks into PMP implementation. A total of 75 survey statement items were used to assess twelve constructs across three CFIR domains using a survey adapted from the Cancer Prevention and Control Research Network for cancer control EBIs with FQHCs [see Additional file 2] ${ }^{33-35}$ A mean score for each clinic was created to measure level of agreement with each statement item $(5=$ completely agree to $1=$ completely disagree). Twenty survey statements were recoded to align with level of agreement and scoring direction (E.g., Question in Additional file 2: It will be hard to train providers and staff to implement the PMP). Onesided t-tests were conducted to analyze mean score changes (mean difference $<0$ ) between the clinic adoption and implementation survey responses. 


\section{Results}

A total of $26 \mathrm{FQHC}$ and charity care clinical locations serving underserved women in the Greater Houston area were approached for study enrollment. Of the 26 clinics, 22 elected to adopt PMP (85\%). Two clinics enrolled (three clinical locations), but did not complete the trial yielding a total of 19 clinical sites for analysis. A total of 4408 total patients were recruited for the study as shown in Figure 1. Six patients were excluded from the final analysis because we were unable to determine their mammography appointment adherence outcome from the Redcap responses. Of the 4402 in the final analysis, 2078 were enrolled in the baseline period and 2324 were enrolled in the intervention period. The results of the descriptive analysis comparing those in the baseline and intervention periods are reported in Table 1. Women aged 45 to 54 years old made up the largest age group in both periods (baseline $-45 \%$; intervention $-41 \%$ ). The patients in the baseline intervention had a higher percentage of mammography attendance in the Summer (baseline - $47 \%$; intervention - 18\%) compared to those intervention period who had a higher attendance in the Spring (baseline - 8\%; intervention - 36\%). Over half of patients in both periods were in wave 1 (baseline - 59\%; intervention - 51\%). Group 3 was largest in the baseline intervention (baseline $41 \%$; intervention $-14 \%$ ) while group 1 was the largest in the intervention period (baseline $-26 \%$; intervention - 69\%). In terms of mobile mammography provider, provider 2 who had no existing reminder call or group education was the largest mobile mammography provider to women in both periods (baseline $-48 \%$; intervention - 52\%). Hispanic women were the largest served racial/ethnicity group across clinics in both periods (baseline $-77 \%$; intervention $-70 \%$ ).

The results of the descriptive analysis comparing those in the intervention period who did and did not complete the intervention are also reported in Table 1. Out of the 2324 patients in the intervention period, a total of 1572 completed the PMP intervention. The characteristics of those who completed the intervention were similar to those who did not complete the intervention in regards to patient age group, season, and mobile mammography provider. In terms of wave and group, the percentage of patients who did not complete the intervention was higher in wave 1 (did not complete - $58 \%$; completed intervention 47\%) while $71 \%$ of those who completed the intervention were in group 1 . With Hispanic women being the largest served racial/ethnicity group in the intervention, a higher percentage of those who did not complete the intervention were from a clinic serving predominately Hispanic women (did not complete $76 \%$; completed intervention $-67 \%$ ). In terms of implementation variables, a higher percentage of those who completed the intervention received a call from a BHCTexas $\mathrm{CHW}$ compared to a clinic $\mathrm{CHW}$ (did not complete $-68 \%$; completed intervention $-74 \%$ ). For those who did not complete the intervention, $38 \%$ answered the reminder call. For those who completed the intervention, $72 \%$ received one reminder phone call compared to $46 \%$ of those who did not complete the intervention. In terms of language, a higher percentage of patients who did not complete the intervention received a call in English (did not complete - 51\%; completed intervention - 39\%) while a higher percentage of those who completed the intervention received the call in Spanish (did not complete $-49 \%$; completed intervention - 53\%). All patients who received a call in Vietnamese completed the intervention. 
In the bivariate analysis, multiple statistically significant differences in mammography appointment adherence were identified as shown in Table 2. A statistically significant difference in appointment adherence was observed between periods $(p<0.05)$, completion of the PMP intervention $(p<0.001)$, wave $(p<0.01)$, group $(p<0.001)$, mobile mammography provider $(p<0.001)$, and clinic racial distribution $(p<0.001)$. A marginal trend toward significance $(p=0.058)$ was observed for both age and season. Those who attended or rescheduled their mammography appointment were more likely to be in the intervention period, complete the intervention, be in wave 1 and group 1, be served by a clinic serving predominately Non- Hispanic women who identified with a race other than Black or white, and be served by mobile mammography provider 1 who had an existing reminder call and group education program compared to those who did not show or cancelled their appointment. In terms of the implementation variables, the number of reminders call attempts, if the patient answered the reminder call, and language of the reminder call were statistically significant $(p<0.001)$. Those who attended or rescheduled their mammography appointment were more likely to answer the reminder call, receive one reminder call, and receive a reminder call in Spanish or Vietnamese compared to those who did not show or cancelled their appointment. Among the 2078 patients in the baseline period, 448 (22\% no-show rate) did not show up to their appointment, whereas among the 2324 patients in the intervention period 438 (19\% no-show rate) did not show up to their appointment. Among the 752 patients who did not complete intervention 205 (27\% no-show rate) did not show up, whereas among the 1572 patients who completed the intervention 233 (15\% no-show rate) did not show up.

Table 3 includes the results of the multivariable GEE logistic regression performed to fit the covariates to mammography appointment adherence by baseline and intervention period and by completion of the intervention. In the first model, the intervention period, relative to the baseline period, was associated with higher odds of attending or rescheduling a mammography appointment $(O R=1.30 ; p<0.01)$. The age group of 25 to 44 years was associated with lower odds compared to the 55 years and older age group $(O R=0.73 ; p<0.001)$. Mobile mammography provider 2 was associated with lower odds $(O R=0.60 ; p<$ 0.01 ) compared to mobile mammography provider 1 . In the second model, completing the intervention, relative to not completing, was associated with higher odds of attending or rescheduling an appointment $(O R=1.62 ; p<0.01)$. As in the first model, the age group of 25 to 44 years was associated with lower odds compared to the 55 years and older age group $(O R=0.71 ; p<0.05)$. Relative to receiving one reminder call, receiving multiple reminder call attempts was also associated with lower odds attending or rescheduling an appointment $(O R=0.78 ; p<0.05)$.

\section{Implementation process evaluation}

A total of 20 clinics completed the adoption survey prior to implementation, with 15 of these 20 clinics ultimately adopting and implementing the PMP intervention. Of those 15 clinics who completed the adoption survey and implemented the PMP intervention, eight clinics completed the implementation survey at eight weeks post implementation in their site. Table 4 includes the results of comparing the clinic mean scores from the adoption survey to the implementation survey. While we observed a statistically significant $(p<0.05)$ decreases in Inner Setting overall and in Culture - Effort and 
Implementation Climate (Inner Setting constructs), potential directional trends can be identified from the results. We observed a decrease in Intervention Characteristic constructs such as Relative Advantage, Trialability, and Compatibility, but an

increase in Complexity (i.e., easier to implement). We observed an increase in the Inner Setting construct of Culture - Stress (i.e., improvements in staff stress and frustration) and in the Outer Setting constructs of Policies and Incentives and Patients Needs and Resources.

The implementation survey included additional questions to assess motivation to participate in PMP, enrollment in PMP, and influence of the PMP adoption webinar. Across the eight clinics, a total of 16 clinic staff members completed these questions. Clinics staff members reported that their motivation to participate in PMP included participating in an EBI, helping patients to understand the importance of mammography screening, and reducing their mammography no-show rates. Staff members reported being more highly motivated to participate in PMP because of the partnership between BHCTexas and the researchers, compared to just their membership in BHCTexas alone. All but one clinic staff member who participated in the webinar reported that the webinar influenced their decision to enroll in PMP. All clinic staff members found the enrollment process for PMP easy.

\section{Discussion}

This study has important implications for the implementation and scaling of EBIs in clinics serving underserved populations and for cancer prevention in underserved women. First, our stepped wedge design allowed us to accommodate readiness to implement PMP in a real-world setting and to proactively engage practitioners in the planning and implementation of PMP. Our CFIR survey results indicate clinics could describe a concrete need for PMP during implementation. Interestingly, no clinics reported being incentivized by the financial stipend provided to the clinic. This has been shown in other studies to be a factor which positively influences implementation. ${ }^{36}$ Our survey also found that our engagement approach in the implementation strategy led to readiness to implement and increased readiness in the baseline survey results (adoption). This approach to stakeholder engagement could serve as a model for other programs and is described in detail in elsewhere. ${ }^{30}$ Previous studies have shown that a lack of engagement with practitioners and implementers has led to programs that do not represent nor fit with the communities they are intended to impact. ${ }^{28}$

Within the cancer realm, a wide gulf between research and practice continues to lead to suboptimal EBI implementation. ${ }^{37}$ We sought specifically to address these gaps in the development of PMP and in partnering with BHCTexas as our network partner in this study. ${ }^{28-29}$ We hypothesized that a communityacademic partnership would positively impact adoption and implementation and serve a bridging role to recruit, motivate and support clinics serving underserved women. Bridging factors in implementation science consider relational ties, strength, processes and formal arrangements that connect the Inner and Outer Setting. ${ }^{38}$ Using a community-academic partnership, focusing on internal and external incentives, addressing funding gaps, and staging implementation were all applied in the PMP implementation 
strategy. ${ }^{28-29}$ Recent research has shown the urgent priority and current gap in the implementation science literature related to bridging and its impact on EBI implementation. ${ }^{38}$ Our CFIR survey indicates this relationship highly motivated clinics to adopt. Our survey also found directional (though not significant) increases in Outer setting constructs related to bridging (Patient Needs and Resources) and in Inner Setting constructs related to ease of implementation (Culture - Stress). We also found statistically significant decreases in some Inner Setting constructs (Culture - Effort and Implementation Climate). It is possible that eight weeks post-implementation was too soon a time period for staff to feel that sufficient systems to support PMP were in place in their clinics and that they had sufficient self-efficacy to lead without support. It also is possible that Inner Setting scores decreased at implementation due to staff gaining a more realistic understanding of what successful EBI implementation takes once being exposed to PMP in daily operations. It is also possible that this change reflects the dynamic nature of working in under-resourced, high stress clinical environments. Our previous work in PMP development and results from this study provide an opportunity to expand the knowledge base related to bridging strategies on EBI implementation, highlight practical approaches that can be replicated or built upon by other implementation scientists and identifies opportunities where further study is warranted (such as the effect on Inner Setting constructs).

Our findings further show that the use of a pragmatic approach to implementation can successfully translate an EBI to practice in underserved clinics. Pragmatically, we implemented across two distinct waves to reach more clinics and women with limited staff. In the GEE model, no statistically significant difference in Wave was observed, indicating that PMP was implemented with fidelity across the trial time periods (two separate enrollments of clinics). This finding could be beneficial for others working in a limited resource environment to maximize reach of EBIs. We also found that standardized training leveled the competencies of staff to implement PMP. Previous studies have shown that inconsistent training and lack of standardized competencies for navigators hinders EBI success. ${ }^{39}$ Our implementation survey collected eight weeks post training found no significant change in Readiness for Change from adoption to implementation, however, each site completed a readiness assessment checklist indicating they were ready to implement PMP prior to program implementation. It is likely the CFIR survey used in this study did not have enough power to detect a difference in this construct, due to clustering of respondents in clinical sites. Our PMP stakeholder engagement and training approach could be adopted and adapted to other cancers in future studies and further tested for its potential impact on readiness. In the intervention period model, no differences in appointment attendance were observed between patients completing PMP with the BHCTexas CHWs (trainers) and the clinic staff (trainees), indicating that PMP training was successful. Second, through our stakeholder engagement process any program adaptations that may have been needed to implement PMP were considered proactively. ${ }^{30}$ In our intervention period GEE model, all patients received the expected multiple phone call attempts, all patients who answered were asked the staging question and received the scripted counseling protocol, which indicates fidelity to active ingredients of PMP. During our implementation survey clinics reported improvements in their perception of program complexity and that PMP was easier than they expected to implement. 
Third, PMP improved the no-show rate across the trial and achieved impact across a diverse patient population, which included Hispanic, non-Hispanic Black, non-Hispanic white, and Vietnamese women. Baseline no-show rates varied by clinic, with an overall average no-show rate in participating clinics of $22 \%$. In the intent to treat analysis, women who were in the intervention period were 1.30 higher odds of attending their appointment than those in the baseline period after controlling for known confounders including age, race, language, season, and mobile provider and the no-show rate reduced to $19 \%(p=0.05)$. In the intervention period, women who fully completed the PMP intervention (received the staging question and barriers counseling) were at 1.62 higher odds of attending their appointments than those who did not complete the intervention, after controlling for known confounders including age, race, language, season, and mobile provider. Eighty five percent of women who completed the PMP EBI attended their mammography appointments $(15 \%$ no-show rate; $p<0.001)$. Previous studies have shown high variability in no-show rates in FQHCs and clinics serving underserved populations, with some sites having no-show rates as high as $45 \% .{ }^{40}$ Our population-averaged baseline no-show rate for participating clinics was lower than previous studies at $22 \%$. Due to our use of a population averaged approach, we may have missed variability within specific clinics or operating sites' no-show rates in this analysis. While our impact in the intent to treat model was a roughly two-percentage point reduction, in patients who completed PMP, no-show rates were reduced by seven percent. The impact of no-shows for mammography screening, breast cancer diagnosis and outcomes cannot be overstated. Women who miss appointments are more likely to never screen, have delayed diagnosis, later stage diagnosis and worse outcomes, including survival. ${ }^{28}$ Additionally, clinics serving underserved populations operate with limited financial margins and no-shows significantly impact their financial solvency. ${ }^{40}$

No differences in attendance were observed by clinic race in the intervention period model indicating that PMP successfully addressed barriers across these diverse population groups. Further, language in which PMP was delivered, which was statistically significant in bivariate analysis, was not significant in the intervention period model. This indicates that PMP delivery was effective in multiple languages. Previous studies have found that interventions delivered in a patient's native language facilitates successful implementation. ${ }^{41-42}$ Marginal trends observed for season disappeared in the intervention period model indicating PMP successfully addressed structural barriers that have been shown to affect patient appointment attendance during the year (such as lack of time off). Effect of mobile provider's usual care reminder and education practices on attendance also disappeared in the GEE model for women who completed our program, indicating that PMP successfully improved appointment adherence to an equivalent level across providers despite this variation in routine care delivery.

Limitations of the study include not randomizing the start dates of clinics, which could have resulted in bias. We evaluated the effect of wave and group in our GEE models and found no impact. Women of younger age did retain lower odds of attendance even when completing PMP, indicating that there may be unique barriers in this age group which may require additional assessment and tailoring. This finding should be investigated in future studies to understand if younger women face unique barriers to appointment adherence. After the U.S. Preventive Services Task Force (USPSTF) changed the 
mammography screening recommendations in 2009, studies found a decrease in screening rates among women under the age of $50 .{ }^{43-45}$ Related to the conflicting recommendations, younger women might experience confusion and anxiety in their decision making to get a mammography. Patients with multiple call attempts were also at lower odds of attending their appointments, highlighting the challenges of reaching this population in the short time period prior to their appointment. Underserved women and patients in FQHCs have been shown to be harder to reach, with higher incidence of disconnected phone numbers. ${ }^{46}$ We also conducted the implementation survey at eight weeks post PMP implementation for each site. There is no literature to indicate the ideal timing for assessment of these constructs once an EBI has been implemented. It is possible that eight weeks was too soon to observe differences, though we did find some were statistically significant and others were directionally correct. The survey was intended to assess implementation moderators of PMP impact in each clinic and was not intended to influence program components during delivery, as these had been previously developed. ${ }^{28}$ The survey was provided to all clinical site staff, though not all completed it despite reminders. Due to low numbers of staff completing the survey in each site, we were not able to perform more advanced statistical analysis. The survey has been previously tested for validity, however, and we believe there is value in sharing our descriptive findings. ${ }^{33-35}$ High rates of staff turnover did occur in the clinics during the study which required ongoing training and support and may impact sustainability of the implementation strategy (e.g., trainings, stakeholder meetings, support) and the PMP intervention. Studies have shown staff turnover to be a challenge in this setting. ${ }^{47-49}$ Despite challenges in reaching patients and with staff turnover, systematic reviews have shown the positive impact of patient navigation interventions in FQHCs. ${ }^{39}$

\section{Conclusions}

The PMP EBI improved mammography appointment adherence across a diverse patient population and led to improvement or directional change in implementation constructs across the domains of Intervention Characteristics, Inner Setting, and Outer Setting in clinics serving underserved women. This study extends the knowledge base in multiple areas and could serve as a model for replication for implementation practitioners.

\section{Declarations}

\section{Ethics approval and consent to participate}

The study received IRB approval from the Committee for the Protection of Human Subjects at The University of Texas Health Science Center at Houston (UTHealth).

\section{Consent for publication}

N/A

\section{Availability of data and material}


The datasets generated and/or analyzed during the current study are not publicly available due identifying clinic and patient information but are available from the corresponding author on reasonable request.

\section{Competing interests}

The authors declare that they have no competing interests.

\section{Funding}

The role of funder, the Agency for Healthcare Research and Quality (AHRQ) (Grant number: 1R18HS023255-01), was to provide financial support for the study.

\section{Authors' contributions}

SR, GW, and LH conceptualized the study. JH led manuscript development and prepared the final manuscript. JH, SR, and JS conducted the data analysis. JH, GF, GW, and LH were contributors to the manuscript writing and revisions. All authors have read and approved the manuscript.

\section{Acknowledgements}

The authors would like to acknowledge the Breast Health Collaborative of Texas (BHCTexas) and participating clinical partners for their ongoing collaboration. The authors would like to acknowledge the project funder, the Agency for Healthcare Research and Quality (AHRQ), for their support.

\section{References}

1. National Cancer Institute. SEER Cancer Stat Facts: Female Breast Cancer. https://seer.cancer.gov/statfacts/html/breast.html (2021). Accessed 30 April 2021.

2. Yedjou CG, Sims JN, Miele L, Noubissi F, Lowe L, Fonseca DD, Alo RA, Payton M, Tchounwou PB. Health and racial disparity in breast cancer. Breast Cancer Metastasis and Drug Resistance. 2019:3149. doi:10.1007/978-3-030-20301-6_3

3. Richardson LC, Henley SJ, Miller JW, Massetti G, Thomas CC. Patterns and trends in age-specific black-white differences in breast cancer incidence and mortality-United States, 1999-2014. Morbidity and Mortality Weekly Report. 2016 Oct 14;65(40):1093-8.

4. Schueler KM, Chu PW, Smith-Bindman R. Factors associated with mammography utilization: a systematic quantitative review of the literature. Journal of women's health. 2008 Nov 1;17(9):147798. https://doi.org/10.1089/jwh.2007.0603

5. Onitilo AA, Engel JM, Liang H, Stankowski RV, Miskowiak DA, Broton M, Doi SA. Mammography utilization: patient characteristics and breast cancer stage at diagnosis. American Journal of Roentgenology. 2013 Nov;201(5):1057-63. https://doi.org/10.2214/ajr.13.10733 
6. Patel K, Kanu M, Liu J, Bond B, Brown E, Williams E, Theriot R, Bailey S, Sanderson M, Hargreaves M. Factors influencing breast cancer screening in low-income African Americans in Tennessee. Journal of community health. 2014 Oct 1;39(5):943-50. https://dx.doi.org/10.1007\%2Fs10900-014-9834-x

7. Sawin EM. "The Body Gives Way, Things Happen": Older women describe breast cancer with a nonsupportive intimate partner. European Journal of Oncology Nursing. 2012 Feb 1;16(1):64-70. https://dx.doi.org/10.1016\%2Fj.ejon.2011.03.006

8. Darden J, Rahbar M, Jezierski L, Li M, Velie E. The measurement of neighborhood socioeconomic characteristics and black and white residential segregation in metropolitan Detroit: Implications for the study of social disparities in health. Annals of the Association of American Geographers. 2010 Jan 1;100(1):137-58. https://doi.org/10.1080/00045600903379042

9. Wang F, McLafferty S, Escamilla V, Luo L. Late-stage breast cancer diagnosis and health care access in Illinois. The Professional Geographer. 2008 Jan 1;60(1):54-69.

https://dx.doi.org/10.1080\%2F00330120701724087

10. Roetzheim RG, Ferrante JM, Lee JH, Chen R, Love-Jackson KM, Gonzalez EC, Fisher KJ, McCarthy EP. Influence of primary care on breast cancer outcomes among Medicare beneficiaries. The Annals of Family Medicine. 2012 Sep 1;10(5):401-11. https://doi.org/10.1370/afm.1398

11. Norman SA, Localio AR, Zhou L, Weber AL, Coates RJ, Malone KE, Bernstein L, Marchbanks PA, Liff JM, Lee NC, Nadel MR. Benefit of screening mammography in reducing the rate of late-stage breast cancer diagnoses (United States). Cancer Causes \& Control. 2006 Sep;17(7):921-9. https://doi.org/10.1007/s10552-006-0029-3

12. Rajan SS, Begley CE, Kim B. Breast cancer stage at diagnosis among medically underserved women screened through the Texas breast and cervical cancer services. Population health management. 2014 Aug 1;17(4):202-10. https://doi.org/10.1089/pop.2013.0079

13. Irvin VL, Zhang Z, Simon MS, et al. Comparison of Mortality Among Participants of Women's Health Initiative Trials with Screening-Detected Breast Cancers vs Interval Breast Cancers. JAMA Netw Open. 2020;3(6):e207227. https://dx.doi.org/10.1001\%2Fjamanetworkopen.2020.7227

14. Duffy SW, Tabár L, Yen AM, Dean PB, Smith RA, Jonsson H, Törnberg S, Chiu SY, Chen SL, Jen GH, Ku MM. Beneficial Effect of Consecutive Screening Mammography Examinations on Mortality from Breast Cancer: A Prospective Study. Radiology. 2021 Mar 2:203935. https://doi.org/10.1148/radiol.2021203935

15. American Cancer Society. American Cancer Society Recommendations for the Early Detection of Breast Cancer. https://www.cancer.org/cancer/breast-cancer/screening-tests-and-earlydetection/american-cancer-society-recommendations-for-the-early-detection-of-breast-cancer.html (2021). Accessed 30 April 2021.

16. Henry KA, Sherman R, Farber S, Cockburn M, Goldberg DW, Stroup AM. The joint effects of census tract poverty and geographic access on late-stage breast cancer diagnosis in 10 US States. Health \& place. 2013 May 1;21:110-21. https://doi.org/10.1016/j.healthplace.2013.01.007 
17. Rajan SS, Begley CE, Highfield LD, Kim B. Survival benefits of treatment access among underserved breast cancer patients diagnosed through the Texas breast and cervical cancer services program. Journal of Public Health Management and Practice. 2015 Sep 1;21(5):477-86. https://doi.org/10.1097/PHH.0000000000000255

18. Thompson B, Hohl SD, Molina Y, Paskett ED, Fisher JL, Baltic RD, Washington CM. Breast cancer disparities among women in underserved communities in the USA. Current breast cancer reports. 2018 Sep;10(3):131-41. https://doi.org/10.1007/s12609-018-0277-8

19. Gathirua-Mwangi W, Cohee A, Tarver WL, Marley A, Biederman E, Stump T, Monahan P, Rawl S, Skinner CS, Champion VL. Factors associated with adherence to mammography screening among insured women differ by income levels. Women's Health Issues. 2018 Sep 1;28(5):462-9. https://doi.org/10.1016/j.whi.2018.06.001

20. Vang S, Margolies LR, Jandorf L. Peer Reviewed: Mobile Mammography Participation Among Medically Underserved Women: A Systematic Review. Preventing chronic disease. 2018;15. https://doi.org/10.5888/pcd15.180291

21. Henderson V, Tossas-Milligan K, Martinez E, Williams B, Torres P, Mannan N, Green L, Thompson B, Winn R, Watson KS. Implementation of an integrated framework for a breast cancer screening and navigation program for women from underresourced communities. Cancer. 2020 May 15;126:248193. https://doi.org/10.1002/cncr.32843

22. Sheppard VB, Wang JH, Eng-Wong J, Martin SH, Hurtado-de-Mendoza A, Luta G. Promoting mammography adherence in underserved women: The telephone coaching adherence study. Contemporary clinical trials. 2013 May 1;35(1):35-42. https://doi.org/10.1016/j.cct.2013.02.005

23. Nonzee NJ, Ragas DM, Ha Luu T, Phisuthikul AM, Tom L, Dong X, Simon MA. Delays in cancer care among low-income minorities despite access. Journal of women's health. 2015 Jun 1;24(6):506-14. https://doi.org/10.1089/jwh.2014.4998

24. Taplin SH, Anhang Price R, Edwards HM, Foster MK, Breslau ES, Chollette V, Prabhu Das I, Clauser SB, Fennell ML, Zapka J. Introduction: understanding and influencing multilevel factors across the cancer care continuum. Journal of the National Cancer Institute Monographs. 2012 May 1;2012(44):2-10. https://doi.org/10.1093/jncimonographs/lgs008

25. Ahmed SM, Palermo AG. Community engagement in research: frameworks for education and peer review. American journal of public health. 2010 Aug;100(8):1380-7.

26. Allen CL, Harris JR, Hannon PA, Parrish AT, Hammerback K, Craft J, Gray B. Opportunities for improving cancer prevention at federally qualified health centers. Journal of Cancer Education. 2014 Mar;29(1):30-7. https://dx.doi.org/10.1007\%2Fs13187-013-0535-4

27. Cialdella-Kam L, Sabado P, Bispeck MK, Silverman S, Bernstein L, Krawiec V, Hawk E, O'Donnell JF. Implementing cancer prevention into clinical practice. Journal of Cancer Education. 2012 May;27(2):136-43. https://dx.doi.org/10.1007\%2Fs13187-012-0331-6

28. Highfield L, Rajan SS, Valerio MA, Walton G, Fernandez ME, Bartholomew LK. A non-randomized controlled stepped wedge trial to evaluate the effectiveness of a multi-level mammography 
intervention in improving appointment adherence in underserved women. Implementation Science. 2015 Dec;10(1):1-8. https://doi.org/10.1186/s13012-015-0334-x

29. Highfield L, Valerio MA, Fernandez ME, Eldridge-Bartholomew LK. Development of an implementation intervention using intervention mapping to increase mammography among low income women. Frontiers in public health. 2018 Oct 26;6:300. https://doi.org/10.3389/fpubh.2018.00300

30. Holcomb J, Ferguson GM, Sun J, Walton GH, Highfield L. Stakeholder Engagement in Adoption, Implementation, and Sustainment of an Evidence-Based Intervention to Increase Mammography Adherence Among Low-Income Women. Journal of Cancer Education. 2021 Mar 22:1-0. https://doi.org/10.1007/s13187-021-01988-

31. Onitilo AA, Liang H, Stankowski RV, Engel JM, Broton M, Doi SA, Miskowiak DA. Geographical and seasonal barriers to mammography services and breast cancer stage at diagnosis. Rural and remote health. 2014 Aug;14(3):180.

32. Tsuruda KM, Bhargava S, Mangerud G, Sagstad S, Hofvind SS. Monthly variation in mammographic screening attendance in Norway. The European Journal of Public Health. 2017 Dec 1;27(6):1095-7. https://doi.org/10.1093/eurpub/ckx137

33. Fernandez ME, Liang S, Jacobs SR, Taplin SH, Weiner BJ. Pressing ahead: developing and testing of new measures in implementation science. InImplementation Science 2015 Dec (Vol. 10, No. 1, pp. 13). BioMed Central. https://doi.org/10.1186/1748-5908-10-S1-A14

34. Fernández ME, Melvin CL, Leeman J, Ribisl KM, Allen JD, Kegler MC, Bastani R, Ory MG, Risendal BC, Hannon PA, Kreuter MW. The cancer prevention and control research network: an interactive systems approach to advancing cancer control implementation research and practice. Cancer Epidemiology and Prevention Biomarkers. 2014 Nov 1;23(11):2512-21. doi: 10.1158/1055-9965.EPI-14-0097

35. Kegler MC, Liang S, Weiner BJ, Tu SP, Friedman DB, Glenn BA, Herrmann AK, Risendal B, Fernandez ME. Measuring constructs of the consolidated framework for implementation research in the context of increasing colorectal cancer screening in federally qualified health center. Health services research. 2018 Dec;53(6):4178-203. https://dx.doi.org/10.1111\%2F1475-6773.13035

36. Varsi C, Ekstedt M, Gammon D, Ruland CM. Using the consolidated framework for implementation research to identify barriers and facilitators for the implementation of an internet-based patientprovider communication service in five settings: a qualitative study. Journal of medical Internet research. 2015;17(11):e262. https://doi.org/10.2196/jmir.5091

37. Rositch AF, Unger-Saldaña K, DeBoer RJ, Ng'ang'a A, Weiner BJ. The role of dissemination and implementation science in global breast cancer control programs: frameworks, methods, and examples. Cancer. 2020 May 15;126:2394-404. https://doi.org/10.1002/cncr.32877

38. Lengnick-Hall R, Stadnick NA, Dickson KS, Moullin JC, Aarons GA. Forms and functions of bridging factors: specifying the dynamic links between outer and inner contexts during implementation and sustainment. Implementation Science. 2021 Dec;16(1):1-3. https://doi.org/10.1186/s13012-02101099-y 
39. Roland Katherine B, Milliken Erin L, Rohan Elizabeth A, Rorie William E, Signes Carmita-Anita C, Young Paul A. Use of community health workers and patient navigators to improve cancer outcomes among patients served by federally qualified health centers: a systematic literature review. Health Equity. 2017 May 1. https://doi.org/10.1089/heq.2017.0001

40. Ruggeri K, Folke T, Benzerga A, Verra S, Büttner C, Steinbeck V, Yee S, Chaiyachati K. Nudging New York: adaptive models and the limits of behavioral interventions to reduce no-shows and health inequalities. BMC health services research. 2020 Dec;20:1-1. https://doi.org/10.1186/s12913-02005097-6

41. Percac-Lima S, Milosavljevic B, Oo SA, Marable D, Bond B. Patient navigation to improve breast cancer screening in Bosnian refugees and immigrants. Journal of immigrant and minority health. 2012 Aug 1;14(4):727-30. doi: 10.1007/s10903-011-9539-5

42. Mojica CM, Almatkyzy G, Morales-Campos D. A cancer education-plus-navigation intervention implemented within a federally qualified health center and community-based settings. Journal of Cancer Education. 2019 Aug 28:1-8. https://doi.org/10.1007/s13187-019-01611-5

43. Wang AT, Fan J, Van Houten HK, Tilburt JC, Stout NK, Montori VM, Shah ND. Impact of the 2009 US Preventive Services Task Force guidelines on screening mammography rates on women in their 40s. PloS one. 2014 Mar 11;9(3):e91399. https://doi.org/10.1371/journal.pone.0091399

44. Qin X, Tangka FK, Guy GP, Howard DH. Mammography rates after the 2009 revision to the United States Preventive Services Task Force breast cancer screening recommendation. Cancer Causes \& Control. 2017 Jan 1;28(1):41-8. doi: 10.1007/s10552-016-0835-1

45. Nelson HD, Weerasinghe R, Wang L, Grunkemeier G. Mammography screening in a large health system following the US Preventive Services Task Force recommendations and the Affordable Care Act. PloS one. 2015 Jun 29;10(6):e0131903. https://doi.org/10.1371/journal.pone.0131903

46. Biola H, Deyo J, Hayes T, Small L, Chaplin J, Pak-Harvey E, Granger T, Jung L, Fitzgerald L, Crowder C, Patel B. Reaching the Hard-to-Reach: Outcomes of the Severe Hypertension Outreach Intervention. American Journal of Preventive Medicine. 2020 Nov 1;59(5):725-32.

https://doi.org/10.1016/j.amepre.2020.05.030

47. Lyson HC, Ackerman S, Lyles C, Schillinger D, Williams P, Gourley G, Gupta R, Handley M, Sarkar U. Redesigning primary care in the safety net: a qualitative analysis of team-based care implementation. InHealthcare 2019 Mar 1 (Vol. 7, No. 1, pp. 22-29). Elsevier. https://doi.org/10.1016/j.hjdsi.2018.09.004

48. Wagner EH, Gupta R, Coleman K. Practice transformation in the safety net medical home initiative: a qualitative look. Medical care. 2014 Nov 1;52:S18-22. doi: 10.1097/MLR.0000000000000196

49. Sugarman JR, Phillips KE, Wagner EH, Coleman K, Abrams MK. The safety net medical home initiative: transforming care for vulnerable populations. Medical care. 2014 Nov 1:S1-0. https://doi.org/10.1097/mlr.0000000000000207

\section{Tables}


Table 1. Descriptive statistics of patient, intervention, clinic, and implementation variables 


\begin{tabular}{|c|c|c|c|c|}
\hline & $\begin{array}{l}\text { All Patient } \\
(\mathrm{N}=4402)\end{array}$ & & $\begin{array}{l}\text { Intervention Perioc } \\
(\mathrm{N}=2324)\end{array}$ & \\
\hline Variable & $\begin{array}{l}\text { Baseline } \\
\text { Period } \\
(n= \\
2078)\end{array}$ & $\begin{array}{l}\text { Intervention } \\
\text { Period } \\
(n=2324)\end{array}$ & $\begin{array}{l}\text { Did not complete } \\
\text { intervention } \\
(n=752)\end{array}$ & $\begin{array}{l}\text { Completed } \\
\text { intervention } \\
(n=1572)\end{array}$ \\
\hline \multicolumn{5}{|l|}{ Patient Variables } \\
\hline \multicolumn{5}{|l|}{ Age category (years) } \\
\hline 25 to 44 & $\begin{array}{l}519 \\
(25.0 \%)\end{array}$ & $\begin{array}{l}549 \\
(23.6 \%)\end{array}$ & $174(23.1 \%)$ & $375(23.9 \%)$ \\
\hline 45 to 54 & $\begin{array}{l}929 \\
(44.7 \%)\end{array}$ & $\begin{array}{l}958 \\
\text { (41.2\%) }\end{array}$ & $317(42.2 \%)$ & $641(40.8 \%)$ \\
\hline 55 and older & $\begin{array}{l}630 \\
(30.3 \%)\end{array}$ & $\begin{array}{l}817 \\
(35.2 \%)\end{array}$ & $261(34.7 \%)$ & $556(35.4 \%)$ \\
\hline \multicolumn{5}{|l|}{ Intervention Variables } \\
\hline \multicolumn{5}{|l|}{ Season (months) } \\
\hline Winter (Jan - March) & $\begin{array}{l}382 \\
(18.4 \%)\end{array}$ & $\begin{array}{l}537 \\
(23.1 \%)\end{array}$ & $186(24.7 \%)$ & $351(22.3 \%)$ \\
\hline Spring (Apr - June) & $\begin{array}{l}172 \\
(8.3 \%)\end{array}$ & $\begin{array}{l}842 \\
(36.2 \%)\end{array}$ & $256(34.0 \%)$ & $586(37.3 \%)$ \\
\hline Summer (July - Sep) & $\begin{array}{l}981 \\
(47.2 \%)\end{array}$ & $\begin{array}{l}414 \\
(17.8 \%)\end{array}$ & $145(19.3 \%)$ & $269(17.1 \%)$ \\
\hline Fall (Oct - Dec) & $\begin{array}{l}543 \\
(26.1 \%)\end{array}$ & $\begin{array}{l}531 \\
(22.9 \%)\end{array}$ & $165(22.0 \%)$ & $366(23.3 \%)$ \\
\hline \multicolumn{5}{|l|}{ Wave } \\
\hline 1 & $\begin{array}{l}1233 \\
(59.3 \%)\end{array}$ & $\begin{array}{l}1180 \\
(50.8 \%)\end{array}$ & $434(57.7 \%)$ & $746(47.5 \%)$ \\
\hline 2 & $\begin{array}{l}845 \\
(40.7 \%)\end{array}$ & $\begin{array}{l}1144 \\
(49.2 \%)\end{array}$ & $318(42.3 \%)$ & $826(52.5 \%)$ \\
\hline \multicolumn{5}{|l|}{ Group } \\
\hline 1 & $\begin{array}{l}543 \\
(26.1 \%)\end{array}$ & $\begin{array}{l}1596 \\
(68.7 \%)\end{array}$ & $484(64.4 \%)$ & $1112(70.7 \%)$ \\
\hline 2 & $\begin{array}{l}676 \\
(32.5 \%)\end{array}$ & $\begin{array}{l}405 \\
(17.4 \%)\end{array}$ & $169(22.5 \%)$ & $236(15.0 \%)$ \\
\hline 3 & $\begin{array}{l}859 \\
(41.3 \%)\end{array}$ & $\begin{array}{l}323 \\
(13.9 \%)\end{array}$ & 99 (13.2\%) & $224(14.3 \%)$ \\
\hline
\end{tabular}


Clinic Variables

Mammography Mobile Provider

\begin{tabular}{|c|c|c|c|c|}
\hline Provider 1 & $\begin{array}{l}919 \\
(44.2 \%)\end{array}$ & $\begin{array}{l}627 \\
(27.0 \%)\end{array}$ & $220(29.3 \%)$ & 407 (25.9\% \\
\hline Provider 2 & $\begin{array}{l}995 \\
(47.9 \%)\end{array}$ & $\begin{array}{l}1218 \\
(52.4 \%)\end{array}$ & 403 (53.6\%) & $815(51.8 \%)$ \\
\hline Provider 3 & $\begin{array}{l}164 \\
(7.9 \%)\end{array}$ & $\begin{array}{l}479 \\
(20.6 \%)\end{array}$ & $129(17.2 \%)$ & $350(22.3 \%)$ \\
\hline
\end{tabular}

Clinic racial distribution ${ }^{a}$

Non-Hispanic Black

168

$(8.1 \%)$

Non-Hispanic white

Non-Hispanic other

Hispanic

Multi-racial/ethnicity group

Implementation Variables

CHW calling patient

Clinic staff

BHCT staff

Answered reminder call

No

Yes

Number of reminder calls

$(n=1852)^{b}$

1 call

Multiple calls ( 2 or 3 calls)

Language of reminder call

$(\mathrm{n}=1817)^{\mathrm{b}}$

English

Spanish

\section{4}

(7.9\%)

108

(5.2\%)

1591

(76.6\%)
$119(5.1 \%) \quad 42(5.6 \%)$

$119(15.8 \%)$

$(17.9 \%)$

$145(6.2 \%) \quad 9(1.2 \%)$

$574(76.3 \%)$

(70.0\%)
77 (4.9\%)

$296(18.8 \%)$

$136(8.7 \%)$

$1053(67.0 \%)$

$10(0.64 \%)$

$47(2.3 \%) \quad 18(0.77 \%) \quad 8(1.1 \%)$

405 (25.8\%)

$1167(74.2 \%)$

$510(67.8 \%)$

$1167(74.2 \%)$

$0(0 \%)$

$1572(100 \%)$

\begin{tabular}{ll}
$465(61.8 \%)$ & $0(0 \%)$ \\
\hline $287(38.2 \%)$ & $1572(100 \%)$
\end{tabular}

-

$908(72.35 \%)$

$347(27.65 \%)$

324 (54.3\%)

$608(39.0 \%)$

$124(50.6 \%)$

$828(53.0 \%)$ 
${ }^{a}$ Non-Hispanic Black, Non-Hispanic other, and Hispanic ${ }^{b}$ Missing data

Table 2. Association of patient, intervention, clinic, and implementation variables with mammography appointment adherence $(\mathrm{N}=$ 4402) 


\begin{tabular}{|c|c|c|c|}
\hline Variable & $\begin{array}{l}\text { No Show or Cancelled } \\
(\mathrm{N}=886)\end{array}$ & $\begin{array}{l}\text { Attended or } \\
\text { Rescheduled } \\
(\mathrm{N}=3516)\end{array}$ & $\begin{array}{l}\mathrm{p} \\
\text { value }\end{array}$ \\
\hline Study period & & & $<0.05$ \\
\hline Baseline period & $448(50.6 \%)$ & $1630(46.5 \%)$ & \\
\hline Intervention period & $438(49.4 \%)$ & $1886(53.6 \%)$ & \\
\hline Intervention period $(n=2324)$ & & & $\begin{array}{l}< \\
0.001\end{array}$ \\
\hline Did not complete intervention & $205(46.8 \%)$ & $547(29.0 \%)$ & \\
\hline Completed intervention & $233(53.2 \%)$ & 1339 (71.0\%) & \\
\hline \multicolumn{4}{|l|}{ Patient Variables } \\
\hline Age category (years) & & & NS \\
\hline 25 to 44 & $239(27.0 \%)$ & $829(23.6 \%)$ & \\
\hline 45 to 54 & $379(42.8 \%)$ & $1508(42.9 \%)$ & \\
\hline 55 and older & $268(30.3 \%)$ & 1179 (33.5\%) & \\
\hline \multicolumn{4}{|l|}{ Intervention Variables } \\
\hline Season (months) & & & NS \\
\hline Winter (Jan - March) & $178(20.1 \%)$ & $741(21.1 \%)$ & \\
\hline Spring (Apr - June) & $202(22.8 \%)$ & $812(23.1 \%)$ & \\
\hline Summer (July - Sep) & $299(33.8 \%)$ & $1096(31.2 \%)$ & \\
\hline Fall (Oct - Dec) & $207(23.4 \%)$ & $867(24.7 \%)$ & \\
\hline Wave & & & $<0.01$ \\
\hline 1 & $450(50.8 \%)$ & $1963(55.8 \%)$ & \\
\hline 2 & $436(49.2 \%)$ & $1553(44.2 \%)$ & \\
\hline Group & & & $\begin{array}{l}<.001 \\
0.00\end{array}$ \\
\hline 1 & $396(44.7 \%)$ & $1743(49.6 \%)$ & \\
\hline 2 & $201(22.7 \%)$ & $880(25.0 \%)$ & \\
\hline 3 & $289(32.6 \%)$ & $893(25.4 \%)$ & \\
\hline Clinic Variables & & & \\
\hline
\end{tabular}




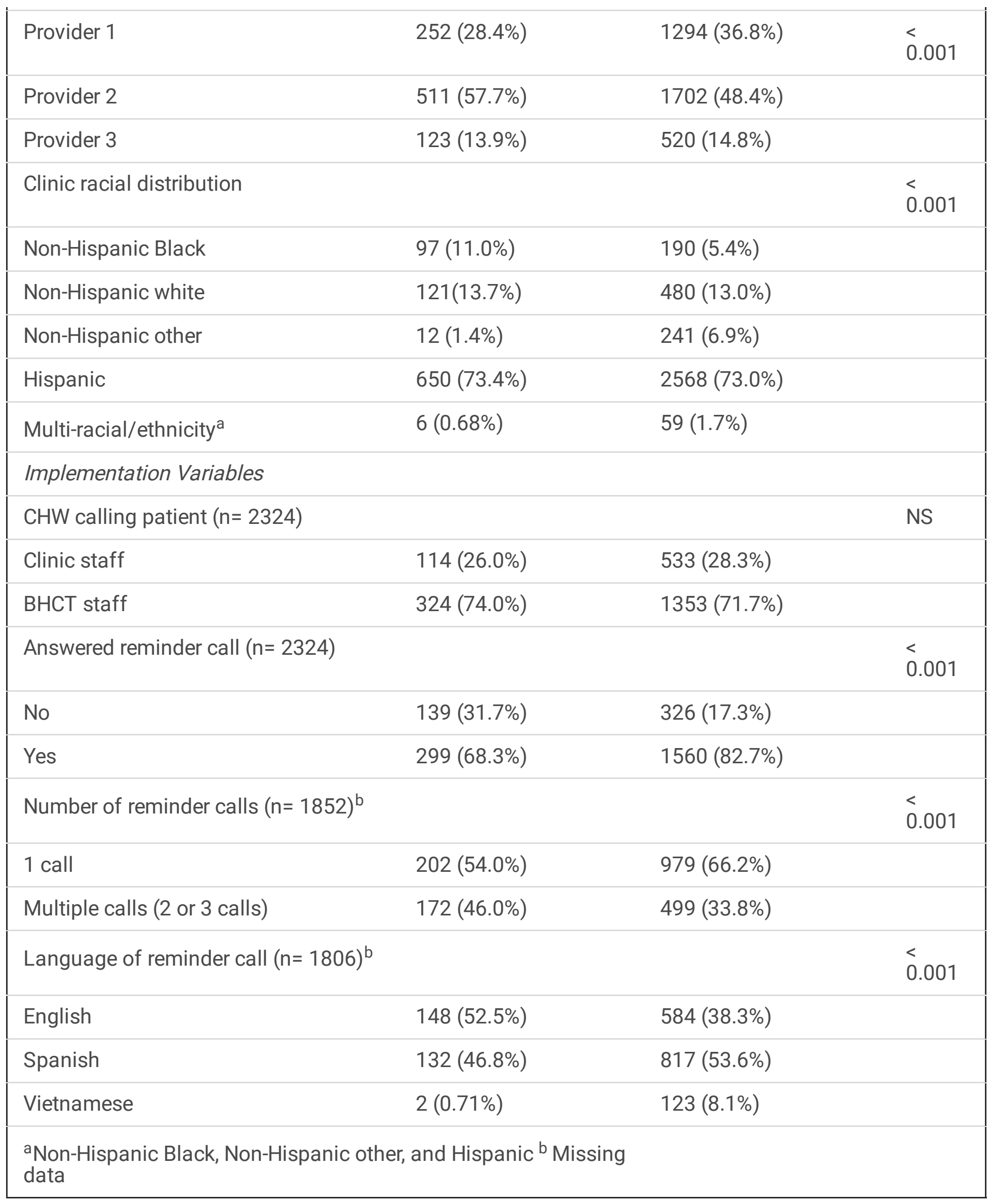


Table 3. Generalized estimating equation (GEE) models assessing mammography appointment adherence by baseline and intervention period and completion of the intervention

\begin{tabular}{|c|c|c|c|c|}
\hline & $\begin{array}{l}\text { Model } 1 \\
(\mathrm{~N}=4402)\end{array}$ & & $\begin{array}{l}\text { Model } 2 \\
(\mathrm{~N}=1852)\end{array}$ & \\
\hline Variable & Odds Ratio & $S E$ & Odds Ratio & $S E$ \\
\hline Study period & $1.30 \star \star$ & 0.12 & - & - \\
\hline Intervention period ${ }^{\mathrm{a}}$ & - & - & $1.62^{\star \star}$ & 0.30 \\
\hline Age category (years) & - & - & & \\
\hline 25 to 44 vs 55 and older & $0.73^{\star \star \star}$ & 0.06 & $0.71 *$ & 0.12 \\
\hline 45 to 54 vs 55 and older & 0.87 & 0.09 & 1.04 & 0.19 \\
\hline \multicolumn{5}{|l|}{ Season (months) } \\
\hline Spring vs Winter & 0.95 & 0.13 & 0.98 & 0.22 \\
\hline Summer vs Winter & 0.95 & 0.14 & 0.84 & 0.25 \\
\hline Fall vs Winter & 1.03 & 0.16 & 1.16 & 0.33 \\
\hline Wave & 0.95 & 0.25 & 0.98 & 0.28 \\
\hline Mobile Mammography Provider 2 & $0.60 \star \star$ & 0.11 & 0.77 & 0.16 \\
\hline Mobile Mammography Provider 3 & 0.63 & 0.2 & 1.09 & 0.35 \\
\hline Clinic racial distribution - Hispanic & 0.82 & 0.37 & 1.52 & 0.58 \\
\hline $\mathrm{CHW}$ calling patient & 0.96 & 0.12 & 0.87 & 0.12 \\
\hline Answered reminder call & - & - & 1.41 & 0.32 \\
\hline Number of reminder calls & - & - & $0.78 *$ & 0.09 \\
\hline $\begin{array}{l}\text { a Did not or did complete the interve } \\
\star p<.05^{\star *} p<.01 * \star * p<.001\end{array}$ & tition & & & \\
\hline
\end{tabular}

Table 4. Descriptive statistics and t-tests for adoption and implementation survey 


\begin{tabular}{|lll|}
\hline & $\begin{array}{l}\text { Adoption } \\
\text { Survey }\end{array}$ & $\begin{array}{l}\text { Implementation } \\
\text { Survey }{ }^{\mathrm{b}}\end{array}$ \\
\hline CFIR ${ }^{\mathrm{a}}$ Domain and Constructs (scale 1 to 5) & Mean (SD) & Mean (SD) \\
\hline Intervention Characteristics & $3.87(0.26)$ & $3.68(0.36)$ \\
\hline Relative advantage & $4.29(0.47)$ & $3.86(0.77)$ \\
\hline Complexity & $3.66(0.35)$ & $3.69(0.39)$ \\
\hline Trialability & $3.24(0.12)$ & $3.18(0.39)$ \\
\hline Compatibility & $4.30(0.33)$ & $4.01(0.48)$ \\
\hline Outer Setting & $3.53(0.25)$ & $3.62(0.39)$ \\
\hline Policies and incentives & $3.52(0.67)$ & $3.56(0.47)$ \\
\hline Patient needs and resources & $3.54(0.24)$ & $3.68(0.15)$ \\
\hline Inner Setting & $3.86(0.27)$ & $3.70(0.33)^{\star}$ \\
\hline Readiness for change / change processes & $3.81(0.25)$ & $3.67(0.35)$ \\
\hline Culture (General) & $3.78(0.18)$ & $3.75(0.21)$ \\
\hline Culture stress & $3.52(0.41)$ & $3.62(0.42)$ \\
\hline Culture effort & $4.06(0.41)$ & $3.92(0.32)^{\star}$ \\
\hline Implementation climate & $4.25(0.38)$ & $3.67(0.82)^{\star}$ \\
\hline Available resources & $3.72(0.29)$ & $3.55(0.55)$ \\
\hline a Consolidated Framework for Implementation Research (CFIR) & \\
\hline b8-weeks post-implementation & & \\
\hline${ }^{\star}$ p <.05; mean(diff) < & & \\
\hline
\end{tabular}

\section{Figures}




\begin{tabular}{|c|c|c|}
\hline \multicolumn{3}{|c|}{ Allocated ( $n=22$ clinics) } \\
\hline Wave 1 & & Wave 2 \\
\hline $\begin{array}{l}\text { Allocated to intervention ( } \mathrm{n}=11 \text { clinics) } \\
\text { Group } 1 \text { ( } \mathrm{n}=3 \text { clinics) } \\
\text { Group } 2(\mathrm{n}=4 \text { clinics) } \\
\text { Group } 3 \text { ( } \mathrm{n}=4 \text { clinics) } \\
\text { Received allocated intervention ( } \mathrm{n}=11 \text { clinics) } \\
\text { Group } 1 \text { ( } \mathrm{n}=3 \text { clinics) } \\
\text { Group } 2(\mathrm{n}=4 \text { clinics) } \\
\text { Group } 3(\mathrm{n}=4 \text { clinics) }\end{array}$ & $\begin{array}{l}\text { Allocated to intervention ( } \mathrm{n}=11 \text { clinics }) \\
\text { Group } 1(\mathrm{n}=5 \text { clinics }) \\
\text { Group } 2(\mathrm{n}=3 \text { clinics }) \\
\text { Group } 3(\mathrm{n}=3 \text { clinics }) \\
\text { Received allocated intervention ( } \mathrm{n}=11 \text { clinics) } \\
\text { Group } 1 \text { ( } \mathrm{n}=5 \text { clinics }) \\
\text { Group } 2(\mathrm{n}=3 \text { clinics }) \\
\text { Group } 3(\mathrm{n}=3 \text { clinics })\end{array}$ & \\
\hline $\begin{array}{l}\text { Discontinued Intervention ( } \mathrm{n}=2 \text { clinics) } \\
\text { Group } 1 \text { ( } \mathrm{n}=0 \text { clinics }) \\
\text { Group } 2(\mathrm{n}=2 \text { clinics }) \\
\text { Group } 3 \text { ( } \mathrm{n}=0 \text { clinics })\end{array}$ & $\begin{array}{l}\text { Discontinued Intervention ( } \mathrm{n}=1 \text { clinic) } \\
\text { Group } 1 \text { ( } \mathrm{n}=1 \text { clinic) } \\
\text { Group } 2 \text { ( } \mathrm{n}=0 \text { clinics }) \\
\text { Group } 3 \text { ( } \mathrm{n}=0 \text { clinics })\end{array}$ & \\
\hline $\begin{array}{l}\text { Analyzed ( } \mathrm{n}=9 \text { clinics, } \mathrm{n}=2,413 \text { patients) } \\
\text { Group } 1 \text { ( } \mathrm{n}=3 \text { clinics, } \mathrm{n}=876 \text { patients) } \\
\text { Group } 2(\mathrm{n}=2 \text { clinics, } \mathrm{n}=701 \text { patients) } \\
\text { Group } 3 \text { ( } \mathrm{n}=4 \text { clinics, } \mathrm{n}=836 \text { patients) } \\
\text { Excluded from analysis, unable to determine } \\
\text { mammography appointment adherence } \\
\text { (n=5 patients) } \\
\text { Group } 1 \text { ( } \mathrm{n}=3 \text { patients) } \\
\text { Group } 2(\mathrm{n}=0 \text { patients) } \\
\text { Group } 3 \text { ( } \mathrm{n}=2 \text { patients) }\end{array}$ & $\begin{array}{l}\text { Analyzed ( } \mathrm{n}=10 \text { clinics, } \mathrm{n}=1,989 \text { patients) } \\
\text { Group } 1 \text { ( } \mathrm{n}=4 \text { clinics, } \mathrm{n}=1,263 \text { patients) } \\
\text { Group } 2 \text { ( } \mathrm{n}=3 \text { clinics, } \mathrm{n}=380 \text { patients) } \\
\text { Group } 3 \text { ( } \mathrm{n}=3 \text { clinics, } \mathrm{n}=346 \text { patients) } \\
\text { Excluded from analysis, unable to determine } \\
\text { mammography appointment adherence } \\
\text { ( } \mathrm{n}=1 \text { patient) } \\
\text { Group } 1 \text { ( } \mathrm{n}=0 \text { patients) } \\
\text { Group } 2 \text { ( } \mathrm{n}=1 \text { patient) } \\
\text { Group } 3 \text { ( } \mathrm{n}=0 \text { patients) }\end{array}$ & \\
\hline
\end{tabular}

\section{Figure 1}

Flow chart showing enrollment, allocation, follow up, and analysis of clinics and patients in the Peace of Mind Program (PMP) study trial

\section{Supplementary Files}

This is a list of supplementary files associated with this preprint. Click to download.

- Additionalfile1.pdf 
- Additionalfile2.pdf

Page 28/28 